\title{
Pulmonary fibrosis: a cellular overreaction or a failure of communication?
}

\section{Commentary}

See related article, pages $1529-1536$.

\author{
Dean Sheppard \\ Department of Medicine, Sandler Center for Basic Research in Asthma and the Lung Biology Center, \\ University of California San Francisco, San Francisco, California, USA
}

Address correspondence to: Dean Sheppard, Lung Biology Center, Box 0854, University of California San Francisco, San Francisco, California 94110, USA. Phone: (415) 206-5901; Fax: (415) 206-4123; E-mail: deans@itsa.ucsf.edu.

Historically, idiopathic pulmonary fibrosis was thought to be a deleterious consequence of persistent lung inflammation that followed an unknown insult. However, the growing recognition that foci of proliferating fibroblasts are more prominent than evidence of inflammation in lung tissue (1), as well as the lack of efficacy of antiinflammatory drugs in the majority of affected patients, has led to a focus on the fibrotic pathway itself (2).

At first blush, the article by Kolb et al. in this issue of the JCI (3) might suggest that we may have discounted inflammation too hastily. The authors show that treatment of rats with a single dose of a recombinant adenovirus encoding the cytokine IL-1 $\beta$, a central regulator of acute inflammation (4), leads to a progressive form of pulmonary fibrosis that continues over at least 60 days. Importantly, this progression is not due to any persistent effects of IL- $1 \beta$ itself, since, as expected with adenovirus infection, IL-1 $\beta$ expression is only increased transiently, returning to near base-line values by 14 days after infection. In contrast, pulmonary fibrosis (as determined by increases in lung hydroxyproline content) is not apparent until day 21 and dramatically increases thereafter. Thus, IL- $1 \beta$ can be added to a short list of agents, including the cytotoxic drug bleomycin (5), ligands of the Fas death receptor (6), and the cytokine TGF- $\beta 1$ (7), that can bypass the normal repair process and initiate a self-perpetuating cycle of pulmonary fibrosis.

Should the article by Kolb et al. (3) rekindle enthusiasm for focusing on acute inflammation in our efforts to better understand the mechanisms underlying diseases characterized by progressive pulmonary fibrosis? I think not. Although severe acute inflammation occurred in response to IL- $1 \beta$, the inflammatory response had resolved by the time fibrosis became apparent and was thus not required for subsequent progression. Although the mechanisms by which IL- $1 \beta$ leads to progressive fibrosis were not clearly defined, the presence of progressive lung fibrosis well after IL-1 $\beta$ concentrations had returned to base line suggests that transient overexpression of this cytokine resulted in dysregulation of processes needed for normal lung repair.

There are at least two possible explanations for the findings in this study. The first is that IL- $1 \beta$ itself somehow reprograms one or more lung cell to continuously produce increased concentrations of profibrotic cytokines, especially TGF- $\beta 1$. This is an attractive hypothesis, since several lines of evidence suggest that TGF- $\beta 1$ is a central regulator of pulmonary fibrosis $(8,9)$. Indeed, since substantial TGF- $\beta 1$ is present in latent form in the lungs of healthy adults, pulmonary fibrosis can result from local activation of the latent cytokine (10) (e.g., through the action of the epithelial integrin $\alpha v \beta 6$ [ref. 11]), even in the absence of any increase in TGF- $\beta$ protein expression.
An alternative explanation might be derived from examination of normal lung development. The mammalian lung depends on a spatially complex and structurally fragile architecture to perform its essential function of gas exchange. Amazingly, this exquisitely precise structure can reform in adults following formidable injuries by microorganisms, environmental insults, or blood-borne toxins. It is thus apparent that the lung microenvironment has a robust capacity to recapitulate at least some of the developmental programs that generated this complex structure in the first place. How then, are components of this developmental process reactivated to effectively repair severely injured lungs of patients recovering from overwhelming bacterial pneumonia or the acute lung injury of sepsis? What fails in the less frequent cases when progressive, fatal lung fibrosis ensues? Herein lie the central issues facing physicians and scientists trying to understand pulmonary fibrosis and to improve methods for treating affected patients.

Although many of the details of normal lung development remain to be fully elucidated, it is quite clear

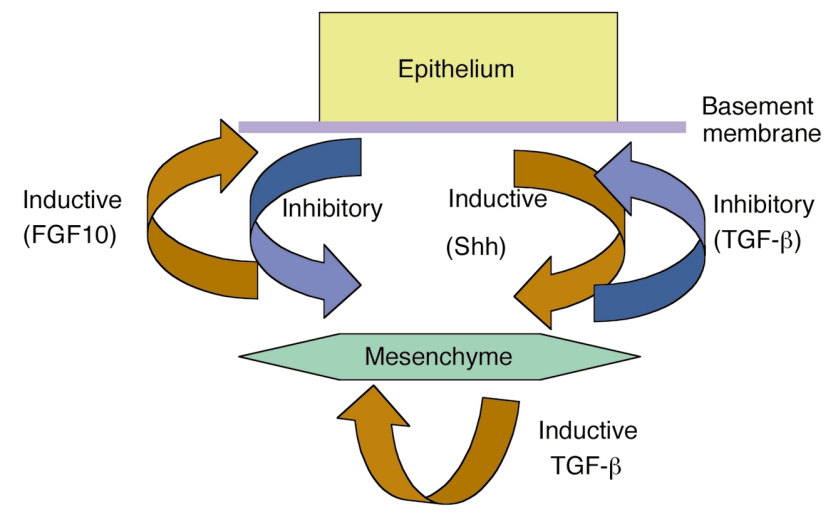

\section{Figure 1}

A few of the inductive and inhibitory signals involved in normal lung development. Some of the same signals are likely to be required for normal lung repair. 
that a central feature is a network of reciprocal inductive and inhibitory signals involving at least mesenchymal cells, pulmonary epithelial cells, and components of the ECM (Figure 1) (12). During development, tight spatial and temporal regulation of this cellular conversation is essential, and the balance of inputs from mesenchyme and epithelium is crucial. Thus, either overexpression or inhibition of some of the critical products of either cell type - e.g., members of the TGF- $\beta$ (13), Hedgehog (14), or FGF families (15) - can dramatically disrupt lung development. In this context, the role of IL-1 $\beta$ in the model described by Kolb et al. (3) could be principally to induce sufficiently severe acute inflammation to cause complete or disproportionate loss of epithelial or mesenchymal cells or of the ECM proteins required to induce normal repair. Indeed, with the exception of TGF- $\beta 1$, each of the stimuli known to cause dramatic and progressive pulmonary fibrosis in rodent models (bleomycin, fas ligation, and IL-1 $\beta$ overexpression) appears to share the ability to cause cell death and loss of structural integrity of alveoli. The remarkable ability of the adult lung to repair itself suggests that substantial loss of alveolar integrity can still allow the normal developmental reciprocal inductive program to proceed. However, this program presumably depends on some threshold or a combination of surviving mesenchymal cells, epithelial cells, and perhaps ECM components. Either death of cells or destruction of the matrix of sufficient scale is likely to perturb this cellular conversation and result in the dysfunctional repair process that characterizes pulmonary fibrosis.

As we evolve a more detailed understanding of which features of normal development are required for normal lung repair, more sophisticated models will emerge. For example, factors that enhance production and/or activation of TGF- $\beta 1$ could tip the balance toward inhibition of epithelial proliferation and enhancement of both fibroblast proliferation and matrix production. In the absence of counterbalancing signals from the epithelium, a positive feedback loop could ensue. This loop would be reinforced both by TGF- $\beta$ inducing its own expression (16) and by its ability to upregulate expression of the $\alpha v \beta 6$ integrin (17), thereby enhancing its own activation. A better understanding of how normal development is regulated and the existence of models of dysregulated development, such as the one described by Kolb et al. (3), are likely to provide fruitful opportunities to improve our understanding of the largely untreatable group of disorders characterized by progressive pulmonary fibrosis.

\section{Acknowledgments}

The author thanks Thiennu Vu, David Morris, and Talmadge E. King, Jr., for helpful comments on the manuscript.

1. Kuhn, C., et al. 1989. An immunohistochemical study of architectural remodeling and connective tissue synthesis in pulmonary fibrosis. Am. Rev. Respir. Dis. 140:1693-1703.

2. Selman, M., King, T.E., Jr., and Pardo, A. 2001 Idiopathic pulmonary fibrosis: prevailing and evolving hypotheses about its pathogenesis and implications for therapy. Ann. Intern. Med. 134:136-151.

3. Kolb, M., Margetts, P.J., Anthony, D.C., Pitossi, F., and Gauldie, J. 2001. Transient expression of
IL-1 $\beta$ induces acute lung injury and chronic repair leading to pulmonary fibrosis. J. Clin. Invest. 107:1529-1536

4. Dinarello, C. 1996. Biological basis for interleukin-1 in disease. Blood. 87:2095-2147.

5. Jones, A.W., and Reeve, N.L. 1978. Ultrastructural study of bleomycin-induced pulmonary changes in mice. J. Pathol. 124:227-233.

6. Hagimoto, N., et al. 1997. Induction of apoptosis and pulmonary fibrosis in mice in response to ligation of fas antigen. Am. J. Respir. Cell Mol. Biol. 17:272-278.

7. Sime, P.J., Xing, Z., Graham, F.L., Csaky, K.G., and Gauldie, J. 1997. Adenovector-mediated gene transfer of active transforming growth factor- $\beta 1$ induces prolonged severe fibrosis in rat lung. $J$. Clin. Invest. 100:768-776.

8. Giri, S.N., Hyde, D.M., and Hollinger, M.A. 1993. Effect of antibody to transforming growth factor $\beta$ on bleomycin-induced accumulation of lung collagen in mice. Thorax. 48:959-966.

9. Phan, S., Gharaee-Kermani, M., Wolber, F., and Ryan, U.S. 1991. Stimulation of rat endothelial cell transforming growth factor-beta production by bleomycin. J. Clin. Invest. 87:148-154.

10. Munger, J.S., et al. 1997. Latent transforming growth factor-beta: structural features and mechanisms of activation. Kidney Int. 51:1376-1382.

11. Munger, J.S., et al. 1999. The integrin $\alpha v \beta 6$ binds and activates latent TGF $\beta 1$ : a mechanism for regulating pulmonary inflammation and fibrosis. Cell. 96:319-328.

12. Warburton, D.M., et al. 2000. The molecular basis of lung morphogenesis. Mech. Dev. 92:55-81.

13. Bellusci, S., Henderson, R., Winnier, G., Oikawa, T., and Hogan, B.L. 1996. Evidence from normal expression and targeted misexpression that bone morphogenetic protein $(\mathrm{Bmp}-4)$ plays a role in mouse embryonic lung morphogenesis. Development. 122:1693-1702.

14. Bellusci, S., et al. 1997. Involvement of Sonic hedgehog (Shh) in mouse embryonic lung growth and morphogenesis. Development. 124:53-63.

15. Peters, K.S., et al. 1994. Targeted expression of a dominant negative FGF receptor blocks branching morphogenesis and epithelial differentiation of the mouse lung. EMBOJ. 13:3296-3301.

16. Van Obberghen-Schilling, E., Roche, N.S., Flanders, K.C., Sporn, M.B., and Roberts, A.B. 1988 Transforming growth factor-beta 1 positively regulates its own expression in normal and transformed cells. J. Biol. Chem. 263:7741-7746.

17. Sheppard, D., Cohen, D.S., Wang, A., and Busk, M. 1992. Transforming growth factor beta differentially regulates expression of integrin sub units in guinea pig airway epithelial cells. J. Biol. Chem. 267:17409-17414. 